Article

\title{
The Visual Discourse of Protest Movements on Twitter: The Case of Hong Kong 2014
}

\author{
Irmgard Wetzstein \\ Department of Communication, Faculty of Social Sciences, University of Vienna, 1090 Vienna, Austria; \\ E-Mail: irmgard.wetzstein@univie.ac.at
}

Submitted: 3 May 2017 | Accepted: 25 September 2017 | Published: 21 December 2017

\begin{abstract}
The article presents the results of a qualitative documentary image interpretation of the visual discourse of the Hong Kong protests on the Twitter hashtag \#hongkongprotests. Visual thematic patterns, the actors depicted, and the relations between actors as well as visual perspectives were analyzed to derive the function of visual images and to give insights into visual protest storytelling. Visuals and image-text relations in Tweets within \#hongkongprotests revealed an application of images in clear favor of the protest movement taking an 'at the scene'/'on the ground' perspective, with media workers being active in front of the camera rather than mere observers behind the camera. While the approach used proved to be suitable for the research project, the research design comes with some limitations, for example in terms of the nongeneralizability of results.
\end{abstract}

\section{Keywords}

documentary image interpretation; Hong Kong; protest movement; social media; Twitter; visual discourse

\section{Issue}

This article is part of the issue "Visual Communication in the Age of Social Media: Conceptual, Theoretical and Methodological Challenges", edited by Uta Russmann (FHWien der WKW University of Applied Sciences for Management \& Communication, Austria) and Jakob Svensson (Malmö University, Sweden).

(C) 2017 by the author; licensee Cogitatio (Lisbon, Portugal). This article is licensed under a Creative Commons Attribution 4.0 International License (CC BY).

\section{Introduction}

Media scholars have been discussing the role of social media, such as Twitter, Facebook and YouTube, in various contexts, including protest movements, with recent studies attesting to social media's positive role in democratic engagement and its impact in supporting and even participating in protest movements such as the civil disobedience movement that took place in Hong Kong from September to December 2014, the focus of the present article (Chen, Chan, \& Lee, 2016; Lee, Chen, \& Chan, 2017; Tufekci, 2017; Zhu, Skoric, \& Shen, 2017). Findings regarding the role of social media and mobile communication in the 2014 Hong Kong protests indicate that young people, as key participants, used their media literacy skills to mobilize, organize, and initiate the protests and collective actions using not only social media and mobile networks but also mass media and street booths, adopting an integrated, holistic approach to disseminating and receiving information (Lee \& Ting, 2015). The Hong Kong protests highlight how digital media activities are positively related to people's involvement in the protests (Lee \& Chan, 2016).

This article assumes social media as assisting supporters to spread the word (Jenkins, Ford, \& Green, 2013) and to call global attention to protest movements visually, when exploring the visual discourse within the Hong Kong protests 2014, using the \#hongkongprotests Twitter hashtag. The Hong Kong protests, also known as Umbrella Movement or Umbrella Revolution, were a civil disobedience movement directed against the decisions of the Chinese Standing Committee of the National People's Congress to reform the Hong Kong electoral system. According to the reforms, only candidates approved by the Chinese government would be allowed to present themselves for election in Hong Kong in the future. In Hong 
Kong, which has the status of a Special Administrative Region and therefore some legal and political autonomy from 'mainland' China, those reforms were widely perceived as restrictions.

The article presents the results of a documentary image interpretation, based on an understanding of visuals as cultural products providing knowledge about the 'world' and generating meaning by making things visible or unseen (Rose, 2012), and a concept of discourse referring to sociology of knowledge, aiming at grasping collective knowledge repertoires, thereby using a broad understanding of knowledge including dailylife theories, internalized everyday practices, stereotypes and routines. The concept of discourse underlying the present study, inter alia, addresses thematic patterns and narrative structures forming meanings and social practices and respective power/knowledge regimes (Keller, 2005, 2013; Wetzstein \& Huber, 2016)-aspects which, as a consequence, appear in the present study's research questions.

\section{Literature Review and Research Questions}

Visual communication researchers have been manifoldly interested in protest movements. For example, based on a content analysis of Romanian politicians' Facebook pages, lonescu (2013) comes to the conclusion that the number of images used positively correlates with the public response rate. Andén-Papadopoulos (2014) establishes a deeper link between images and emerging modes of civic engagement when protesters use the mobile camera phone to record and distribute their own repression through social networks. Ekstrand and Silver (2014) emphasize the dialogic character of images especially in mobile contexts, and, dealing with mobile visual communication as well, Villi (2007) highlights perpetual visual contact as a capacity of the ubiquitous camera phone.

Focusing on the different ways that different communities interpreted a video circulated over mainstream and social media showing police violence against a Hong Kong protester, Jones and Neville Chi Hang (2016) emphasize the relevance of (moving) visual images in protest movements when arguing that 'such videos serve both as evidence of police abuses and discursive artefacts around which viewers construct bodies of shared knowledge, attitudes and beliefs regarding events by engaging in exercises of 'collective seeing" (p. 567). In her ethnographic and visual-analytical research on camera phone practices in Hong Kong and referring to earlier vernacular image making, Grace (2014) discusses the role of the visual image in producing historical memory and focuses on images in the context of camera phones as being new practices of creativity and subjectivity distributed via social media, therewith becoming a form of everyday communication. Moreover, Grace (2014) points out Hong Kong as being the ideal place from which to study the significance of the relatively re- cent and unprecedented avalanche of images in popular culture because of the particularly high use of mobile media' (p. 12) and explains that protest movements are not a new phenomenon in the city due to the wide wealth gap and income inequalities among its population.

Without doubt, all of those studies clearly highlight the importance of visual images in social media and mobile communication in context to protest movements and civic engagement. The present study aims at providing deeper insights into the visual discourse of the 2014 Hong Kong protests in social media, in particular on the micro-blogging platform Twitter, in order to derive visual discursive strategies and the possible functioning of images posted on social media in context of protest movements, based on an in-depth study of visuals linked to the intensively used \#hongkongprotests Twitter hashtag during the 2014 democratization protests in Hong Kong, focusing the following research questions derived from a perspective to discourse in the sociology of knowledge as described above:

RQ1: How is the discourse of the Hong Kong protests organized on Twitter?

RQ1a: Which thematic patterns are narrated?

This research question focuses on the detection of visual protest narratives. Thematic patterns refer to highlighted topics within each visual and accompanying (written) verbal text. Visual and verbal contents were therewith approached as mutually dependent in terms of forming the message.

RQ1b: Which actors are depicted visually? What relations are visible among the identified actors?

RQ1b takes a deeper look at the visuals of interest. When identifying depicted actors by name (e.g. certain protest leaders or politicians) and/or role (e.g. a group of protesters, policemen) accompanying verbal text was used whenever it provided useful context information.

RQ1c: Which visual perspectives are used in which context?

The term visual perspectives refers to the camera angle and subject-camera distance. Regarding camera angle, the present study distinguishes low-angle shot, neutral (eye-level) shot, high-angle shot, the bird's-eye view and oblique view. The subject-camera distance and position of the camera towards actors within the image frame refers to either long shots or close-ups. Referring to film studies, both, camera angles and subject-camera distance, promise to give further insights into the characterization of the protests in general and the actors in particular: While low-angle shots might be used to demonstrate power and a higher level of hierarchy, neutral shots might convey a participation in the scene to the viewer. High-angle shots might help to include a large number of actors, and the bird's-eye view and especially top shots suggest collectivity, while an oblique view might give the image contents more dynamism or con- 
vey the instability of the situation. Regarding the subjectcamera distance, the differentiation between long shots and close-ups might suggest the level of proximity to, accessibility of and familiarity with the actors (the latter especially when there is direct eye-contact with the camera and the observer respectively) from the image observers' point of view (Kress \& van Leeuwen, 1996; Sikov, 2010).

RQ2: What information can be derived from image sources and Twitter users and used Twitter accounts respectively?

Any analysis focusing on visuals must not ignore the producers of the images and those distributing those visuals. Therefore, as far as possible, RQ2 identifies image sources (as far as credits are given) and discovers more about Twitter users and accounts using those images.

RQ3: Which strategies are used to (de-)legitimize the protest movement in general as well as the protesters' and authorities' actions?

Drawing on the insights based on the previous research questions, this research question investigates how the protest movement is depicted, whether in a favorable or unfavorable light.

These questions clearly reflect a sociological (and not linguistic) research interest, with the concept of discourse linked to sociology of knowledge asking for (visually) conveyed social practices and systems of thought: Keller (2011) traces back the concept of discourse in the sociology of knowledge to Foucault's (1972) understanding of discourses as 'historically situated 'real' social practices, not representing external objects but constituting them' (Keller, 2011, p. 46) which implies analyzing the way discourses are formed and structured and how they are 'structuring knowledge domains' (p. 47), through the use of concrete data.

\section{Method and Sampling Approach}

The documentary image interpretation as described by Bohnsack (2009) promised to form a suitable analytical framework to elaborate on the above-mentioned research questions. Using sociology of knowledge as a fundament and therewith following the discourse in the sociology of knowledge applied for the present study, it addresses sociological rather than linguistic perspectives, on which multimodal (discourse) analysis approaches are mostly based (see for example Kress \& van Leeuwen, 1996, 2001; Machin \& Mayr, 2012; Royce \& Bowcher, 2007). It emphasizes power practices-related discursive construction of reality and collective knowledge repertoires as an empirical process and combines qualitative and interpretive research perspectives. Moreover, documentary image interpretation provides a clearly structured toolkit covering all aspects of the research interest as condensed in the research questions. Concretely, the approach consists of a formulating interpretation of each visual under study as a first step, consisting of a pre-iconographic layer (description of motifs, phenomena, objects/subjects) and an iconographic layer (identifiable activities/plots), therewith especially informing RQ1a. After the formulating interpretation, a reflecting interpretation follows as a second step, containing the analysis of the formal composition of each visual (visual perspective taken from observers' view, linked to RQ1c; planimetric composition of images and scenic choreography, linked to RQ1b) as well as an iconologicaliconic interpretation (detecting behavioral perspectives and social relations, linked to RQ2 and RQ3). As Bohnsack (2009) suggests, (written) verbal texts accompanying visual elements were included in the analysis only after image interpretation.

The explained method approach promised an indepth and thorough detection of visual patterns forming the discourse of interest, namely the anti-governmental democratization protests which were demanding universal suffrage and which took place in Hong Kong from September 26 until December 15, 2014, on the Twitter hashtag \#hongkongprotests within which a multitude of visual-containing Tweets are tagged. The Hong Kong protests constitute a good case example when focusing on protest movements in the realm of social media, not only due to the high penetration rate of (camera phone/mobile) technologies and the protest 'tradition' in the city (both highlighted by Grace, 2014 as mentioned above), but also because the protests happened quite recently, and because protesters were mainly young people and students belonging to a generation who grew up with digital media, who are active and savvy in using social media and who have a high affinity to mobile communication, as emphasized by Lee and Ting (2015) and, for example, addressed in link 1 (see annex), taken from the analysis corpus.

While a multitude of Twitter hashtags were (often simultaneously) used when addressing the civil disobedience movement in Hong Kong, the analysis material only includes still images within the widely used Twitter hashtag \#hongkongprotests. This is due not only to research pragmatic reasons (referring to the quantity of material) but also because \#hongkongprotests is a more general description than the widely used labels such as \#occupycentral, \#umbrellamovement or \#umbrellarevolution, and, to give an example, not everybody might have known that the so-called Umbrella Movement evolved from Occupy Central. The analyzed hashtag might have been used more frequently by tourists, less informed people as well as by journalists and non-residents in addition to the other previously mentioned hashtags, which of course might have influenced the visual content in this hashtag.

Moving images were excluded from this study because they would need an analysis approach beyond still images due to the manifold modes to be considered additional to the (written) verbal and visual mode (especially movement, sound, and speech). Lead images serving as teasers or marketing while linked to external websites, mostly to journalistic articles within news media or- 
ganizations' web appearances, were excluded from the analysis material as well. This is because visual logics of traditional news media outlets probably provide a different way of storytelling and therewith perspective on protests than the visual discourse taking place directly on Twitter, for example in terms of elite-orientation which researchers have identified in mainstream news media's political conflict coverage (Ottosen, 2007; Wetzstein, 2011). Even though conducting a full survey of all still images linked to \#hongkongprotests was not feasible due to their sheer quantity, the study aimed at gaining knowledge to answer the research questions and derive the functioning of images posted on social media within protest movements as comprehensively as possible. The material selection and collection aimed at creating a most thorough analysis corpus in terms of image contents and perspectives, making sure that each visual was screened in terms of sample selection. The manual material selection helped to make an initial assessment of visual and verbal contents, actors and visual thematic patterns. Repeatedly occurring image content was collected and analyzed only once. Repeatedly occurring image content was referred to as (1) taking the same visual perspective, (2) depicting the same actors (either the same individuals or the same actor group, such as policemen or a group of protesters), and (3) not contributing new insights regarding the verbal context. This approach of material collection and selection resulted in an analysis corpus consisting of 1,521 images linked to Tweets marked with \#hongkongprotests which underwent the research process as described above.

\section{Results}

A first overview of the research material had already suggested that different visual perspectives are taken in photographs, where the depiction of masses of people from above (also top shots with the use of drones) or from an oblique angle and elevated position (taken for example from hotel rooms) as well as individual or groups of protesters from the front seem to be dominant. Photographs are the most common image type in \#hongkongprotests and tend to function inter alia as 'recorders' of slogans and messages from banners set up by activists. Images generally legitimize the movement, for example when using them to demonstrate disproportionate actions of the police against protesters. The following sections will go into the results according to the research questions mentioned above. Exemplary hyperlinks to Tweets in the annex illustrate the results.

\subsection{Thematic Patterns (RQ1a)}

Telling a story visually is more than converting verbal text to images. In contrast to verbal text and their argumentative logic, visuals are processed associatively and not in a linear way. Recurring motives can form a story by establishing visual thematic patterns (Fuchs, 2015).
Thematic patterns widely refer to the characterization of the protesters and the protest movement within \#hongkongprotests. Visually, medium shots with a neutral camera angle make the protesters appear approachable and familiar. Verbally, they are described in a way that implicitly attributes them as smart, polite, peaceful, educated, decent, responsible, innocent and harmless and even weak when facing armed officials such as the police (see for example links 2-5).

The study also revealed a clear focus on the dimension and size of the protests by depicting masses of people at the protest sites using a high angle and bird'seye view, as a rule, accompanied by written text expressing appreciation for the protesters and their commitment to democracy (links 6-8). Additionally, visuals focus on the protest sites and camps, referring especially to the organization of the occupied space. Tents built in a clear structure are as prevalent motives as barricades and uniformed police standing in a row like 'human barricades' (links 9-13). Those motives convey an impression of the protests sites as being clearly arranged and organized regarding the individuals involved in the protests (mainly protesters and police) as well as the protest camp's infrastructure. Regarding the latter, the protest camps are depicted as living spaces, more concretely as spaces in which protesters are able to go about their daily routines, such as studying (link 4) but also playing cards, doing yoga or even getting married (link 14), the latter prodding to a certain romanticization of the protests in their visual depiction. The multitude of photographs of banners and installations produced by protesters at the protest sites and drawings and graphics produced by protest supporters give the protest movement the notion of being artistic, creative, colorful and (new) media affine, giving references to computer technology (links 15-17).

Visual branding and identity-building of the movement started in late September with banners and installations at or near the project sites (such as the Lennon Wall or the Lion Rock installation) as well as drawings and cartoons using protest symbols-such as the yellow umbrella (used first as a protection tool against tear gas before becoming a symbol of resistance) as well as the yellow ribbon-posted on Twitter by protest supporters. The manifold protest artwork and symbols, often showing clear references to popular culture, are repeatedly depicted throughout the analyzed visual material, using the color yellow as the as the main color representing the protests, while red is used to represent the Chinese government (links 18-27).

Besides the reference to the creative productivity of the protests, the visual contents of \#hongkongprotests revealed the uneven power distribution between the police and the protesters as another dominant thematic pattern emerging from the outset and re-occurring throughout the visual discourse (explained in more detail in the 'actors and relations among actors' section). While the visual discourse within \#hongkongprotests is 
less intense from November 2014 (the second months of the protests), the images in the Twitter hashtag become more colorful. Tents in various colors and protest artwork become main motives with protesters being depicted mainly in the background, if at all. When, for example, tourists and visitors share photographs of tents and artwork as well as photographs of themselves or selfies at the protest camps, they are showing their support and visually referring to the protest camps as being a 'must see' for visitors and tourists (link 28). While its camps remain the main 'scenes' of the protest movement in November, the visual story also considers other places and events outside of the actual protest sites, such as groups of people at European or US universities, lecture rooms providing academic discussion forums regarding the Hong Kong protests, sights in other cities decorated with protest symbols, and solidarity of the Ferguson protesters with the movement in Hong Kong, as a reference to geographically widespread support, and suggesting an expansion of the movement beyond the original protest sites (links 29-31).

Photographs also capture media workers' TV reporting activities at the protest sites and show whole newspaper articles or facsimiles addressing, for example, news coverage of the movement for the purpose of information distribution, or governmental media censorship in China (links 32 and 33). From December 11, shortly before the end of the protests on December 15, photographs document protesters but also reporters packing their things and the police clearing protest camps. Users also use photographs to contrast the protest sites during the protests and afterwards, referring to the absence of the protest camps and a return to 'normality', when documenting street traffic where the protest camps had been (links 34 and 35).

\subsection{Actors and Relations among Actors (RQ1b)}

Staying mainly at the protest sites, the visual discourse at hand is hardly oriented on political elites but widely focuses the pro-democracy protesters, takes advocacy for them and generally addresses the Hong Kong protests as a democracy movement of the many. At the beginning of the protests some protesters are verbally addressed as protest leaders, and mainly the 17-year-old founder of the Hong Kong student activist group 'Scholarism' Joshua Wong ${ }^{1}$ is addressed as being an initiator, spokesperson, and promoter of the protests throughout the discourse (links 36 and 37). However, the visual depiction of protesters remains diverse suggesting that Wong is part of the movement rather than representing it as a whole.

Besides the protesters, the police form a second main actor being visually positioned mostly as the protesters' adversaries. In fact, police-protester relations widely characterize and further the visual storyline of \#hongkongprotests. The visual depiction of protesterpolice encounters suggests a rather unstable relationship, switching between neutral to friendly and conflicting with physical confrontations and the police using violence against protesters. Clashes or confrontations between protesters and the police became main visual motives whenever police were going to or were attempting to clear one of the three protest sites and particularly became a repeated motive when police cleared the Mongkok protest camp at the end of November, with protesters occurring either as individuals whom police used violence against, or as a 'front' of many with several umbrellas (links 38-42).

While the police are also visualized positively and characterized as friendly in less conflictual phases of the protests, the opposition between both groups remains visible with the main motive of police being depicted as a group of uniformed people statically standing in a row in front of protesters, therewith acting as human barricades or walls blocking the protesters (links 10-13). Protesters are visualized behaving politely or even supportive towards the police, for example when a protester protected a policeman from rain with an umbrella (link 16). The visual communication of uneven power distribution and disproportionate power use by the police was one main theme established and continued to be so throughout \#hongkongprotests which brings to mind the David-Goliath frame as a common pattern in journalistic coverage when describing and visualizing conflict events and opponents (Dobernig, Lobinger, \& Wetzstein, 2010; Wetzstein, 2011).

While the visual discourse remains traditional in terms of its narration of protester-police relations, it is not traditional when depicting media workers, mainly photographers and TV journalists, as being active actors rather than simply passive observers behind the cameras who are not normally depicted in the events themselves. With professional photographers and journalists being present in photographs while doing their work, they become part of the visual discourse, which consequently allows the observation of those who normally observe, therewith widening the 'traditional' visual frame and giving an impression of their heavy presence and space occupance in protest movements and other conflict events. Within the visual depiction of media workers in \#hongkongprotests, they either occur as central individual actors when 'proving' via Twitter that they are at the scene or announcing TV coverage of the protests, or within a large group of people capturing protest situations or events (links 43-45).

From November onwards, protest movement opponents started occurring in the visual discourse, although only sporadically and mostly in the (verbally mentioned) context of them disturbing the peaceful protests (link 46) or in the context of the depiction of an 'anti-occupier flotilla' consisting of boats with blue banners saying 'Go

\footnotetext{
1 Joshua Wong and two other young protest leaders were given several months sentences for their roles in the Hong Kong protests only in August 2017 (Phillips, 2017, August 17).
} 
Home' (link 47). Pro-government voices were generally rare within \#hongkongprotests. A reason for this was surely the blocking of Twitter in 'mainland' China while the micro-blogging service was freely accessible in Hong Kong. Political elites and events, such as Barack Obama's visit to Beijing in November, remained side issues within \#hongkongprotests, while civil society actors and their visual support of the movement were dominant besides police-protester relations, which depicted, for example, laughing children at protest sites as well as babies or locations in other cities alongside protest symbols such as yellow ribbons. In addition, the protest camps seem to become sights in and of themselves, attracting visitors and tourists making selfies or taking pictures at and of the protest camps with its manifold artwork, installations, and banners, which helps the protesters to mediate and communicate their claims, demands and attitude (links 15-28). The same goes for protest symbols and tools, mainly the yellow umbrella, visualized not only as a symbol of resistance but also as a protection tool against armed police, with 'weapons versus umbrellas' again emphasizing the uneven power distribution between police and protesters (link 41).

\subsection{Visual Perspectives (RQ1C)}

Though visual perspectives were briefly mentioned in the context of thematic patterns and actors' depictions, they are worth focusing on more explicitly as they create the mise-en-scene of image contents (Sikov, 2010). First of all, it is striking that clashes between protesters and the police are mostly depicted oblique, often in combination with an elevated position (link 41). This perspective certainly reinforces the dynamic character of such events and supports a spontaneous and authentic impression of the image. Masses or large groups of protesters as well as protest sites are generally depicted from bird's eye view or even as a top shot (links 6-8), giving an overview of the whole scene, illustrating the large size, collectivity, and possible impact and power of the movement. Images taking a neutral on-the-ground angle, shared for example by reporters or visitors at a protest site, convey a 'being directly at the scene' and aim to document current events or the protest camps, while close shots are used to portray protesters, revealing their character, seeing them as individuals and focusing on the physical consequences of police brutality (links 2, 3, 39, and 40). While the photographs, in general, seem to provide an authentic depiction of the movement, some seem to be trying to create iconic moments with colors obviously edited in photographic post-production or through the depiction of apparently 'staged' situations (links 54-56).

\subsection{Image Sources, Twitter Users, and Strategies of (De-)Legitimation (RQs 2 and 3)}

Even though only a little can be said about image producers and authors of the Tweets linked to \#hongkong- protests mainly as a result of missing source credits and several re-tweets not being individually traceable due to the large size of the analysis corpus, it becomes clear that many of the image producers are professional photographers and journalists working either independently or for media outlets and news organizations, who often share their visual material on Twitter themselves. Images were also shared and re-tweeted by protesters and individual supporters, including tourists and visitors to the protest sites, as well as with accounts of activist groups, online media services, and academic institutions. Verbal hate speech against the protest movement came from one single Twitter account, mainly re-tweeting photographs of people, allegedly tourists or visitors, with the accompanying verbal text carrying an insulting tone.

Support, sympathy for the movement, and empathy for the protesters are, however, clearly the main narrative within the visual discourse. Therefore, the direction towards the legitimation of the protest movement is obvious while the de-legitimation of the movement as a possible competing narrative approach is nonexistent. Strategies used to visually legitimize the Hong Kong protests include the positive characterization of the protests and protesters and the visual narration of power contrasts between protesters and police, as previously illustrated. The Hong Kong protests have also been positively characterized through 'visual comparisons', such as the example where a burning vehicle linked to the 'London riots' was placed next to an image of well-sorted bags linked to the Hong Kong protests, therewith making a distinction between 'good', constructive and well-behaved, and 'bad', destructive and rioting protests and protesters (link 48). Occasionally, users also express concerns about possible negative consequences for protesters caused by the Chinese government, when for example the 2014 movement was visually linked to the 1989 Tiananmen Square protests in Beijing, which resulted in what is known as the Tiananmen Square Massacre (link 49).

While photographs are the most used image type within \#hongkongprotests, the role of memes, infographics, and drawings cannot not be ignored as they were also used to serve either information and campaigning purposes as well as to spread messages and make fun of domestic political elites. The visual de-legitimation of political elites by their objectification is also observed within the artwork and installations at the protest sites, as has been captured photographically (links 50-53).

\section{Discussion}

The function of the images in \#hongkongprotests is to suggest authenticity, the images capture moments spontaneously on the ground, communicating 'this is happening right now'. Similarly, Vis, Parry, Manyukhina and Evans (2013) address the presentation of what is happening right now within their analysis of images shared on Twitter during the 2011 UK riots, regarding still images 
created from live TV and posted on Twitter as screenshots. Due to its potential for real-time photo sharing Twitter surely constitutes a supportive environment for this immediacy and perceived authenticity. Yet, as identified from the visual discourse of interest, images can also create iconic moments and can alternatively have an artistic rather than a spontaneous or authentic character, therewith providing a good example of how image making as a creative action can enter everyday communication when images are distributed via social media, as Grace (2014) suggests.

In terms of image functioning, what Grace (2014) calls the production of historical memory can be understood as an informative or documenting image function. That is for example, when newspaper articles and other written information material are photographed, or when protesters and supporters use graphics to inform about developments of the movement, and when certain events, such as in context of police violence, are captured and framed accordingly using verbal text. Images illustrating verbal text and dramaturgic image-text relations building up tension or loosening up verbal texts, as known from journalistic contexts (Holicki, 1993), occur as well, when for example using images of injured people to illustrate police violence, or protest symbols without referring to certain events or situations, such as yellow umbrellas in front of skyscrapers (see for example link 26).

The visual discourse of \#hongkongprotests also brought to light a clear use of images in favor of the protest movement, legitimizing and supporting it, consequently revealing a general supportive image function. This supportive image function is not only obvious in image-text relations, but also in image-image relations within Tweets using a visual comparison strategy, when for example, comparing protesters in Hong Kong to protesters in London or contrasting the uneven power distribution between protesters and the police (see for example links 3 and 48). Images in \#hongkongprotests largely take an 'at the scene' and 'on the ground' perspective when mainly centering on protest sites, protesters, as well as events at the protest camps. With a clear supportive function and an 'on the ground' perspective, visual protest storytelling on Twitter obviously follows an approach of empowering the protesters.

\section{Conclusion}

The employed documentary image interpretation gave insights into the supportive character of visual protest storytelling and image functioning in the \#hongkongprotests Twitter hashtag. Due to the non-generalizability of the results, the present results certainly need to be challenged in future research dealing with the visual discourse of other pro-democracy protest movements as well as within other Twitter hashtags or even social media services. Furthermore, future research should challenge the present findings by interviewing protesters, focus on the protest artwork itself and assess the possible cultural implications such as the influence of a 'Western lens' when performing documentary image interpretation. Future research should also scrutinize the reinforcement of social capital through visual protest discourses in social media, compare visual representations among non-journalist Twitter accounts and media journalist accounts, and give more emphasis to the different roles of different image types (such as drawings, cartoons, memes, photographs) in communicating protest movements. Finally, while automated monitoring tools are not (yet) sophisticated enough to reliably grasp qualitative visual contexts (Geise, Rössler, \& Kruschinski, 2016), a quantitative approach possibly supported by automated tools providing simple real-time statistics and historical data (with the latter being quite cost-intensive, however) certainly has potential. Nevertheless, with the qualitative method used in this study focusing particularly on visual images, solid steps were made in grasping the visual discourse of the \#hongkongprotests and respective image functioning on Twitter, providing helpful data for future studies examining, for example, other hashtags.

\section{Acknowledgements}

Open access funding provided by University of Vienna.

\section{Conflict of Interests}

The author declares no conflict of interests.

\section{References}

Andén-Papadopoulos, K. (2014). Citizen camera-witnessing: Embodied political dissent in the age of 'mediated mass self-communication'. New Media \& Society, 16(5), 753-769.

Bohnsack, R. (2009). Qualitative Bild- und Videointerpretation. Einführung in die dokumentarische Methode [Qualitative image and video interpretation. Introduction to the documentary method]. Opladen and Farmington Hills: Verlag Barbara Budrich.

Chen, H., Chan, M., \& Lee, F. F. (2016). Social media use and democratic engagement: A comparative study of Hong Kong, Taiwan, and China. Chinese Journal of Communication, 9(4), 348-366.

Dobernig, K., Lobinger, K., \& Wetzstein, I. (2010). Covering conflict: Differences in visual and verbal coverage on the Gaza-crisis 2009 in four weekly news media. Journal of Visual Literacy, 29(1), 88-105.

Ekstrand, V. S., \& Silver, D. (2014). Remixing, reposting, and reblogging: Digital media, theories of the image, and copyright law. Visual Communication Quarterly, 21(2), 96-105.

Foucault, M. (1972). The archeology of knowledge and the discourse on language. New York, NY: Pantheon Books.

Fuchs, W. T. (2015). Warum das Gehirn Geschichten liebt. Mit Storytelling Menschen gewinnen und überzeugen 
[Why the brain loves stories. Embracing and convincing people with storytelling]. Freiburg: Haufe.

Geise, S., Rössler, P., \& Kruschinski, S. (2016). Automatisierte Analyse medialer Bildinhalte. Potenziale, Grenzen, methodisch-technischer Status Quo und zukünftige Herausforderungen. Eine Bestandsaufnahme [Automated analysis of visual media contents. Potentials, limitations, method-technical status quo and future challenges. A survey]. Medien \& Kommunikationswissenschaft, 64(2), 244-269.

Grace, H. (2014). Culture, aesthetics and affect in ubiquitous media: The prosaic image. London: Routledge.

Holicki, S. (1993). Pressefoto und Pressetext im Wirkungsvergleich: Eine experimentelle Untersuchung am Beispiel von Politikerdarstellungen [Comparing the effect of press photo and press text. An experimental study using presentations of politicians]. Munich: Verlag Reinhard Fischer.

Ionescu, N. (2013). Online political communication: The role of image upload on Facebook. Romanian Journal of Journalism \& Communication/Revista Romana De Jurnalism Si Comunicare-RRJC, 8(1), 53-58.

Jenkins, H., Ford, S., \& Green, J. (2013). Spreadable media. Creating value and meaning in a networked culture. New York, NY: New York University Press.

Jones, R. H., \& Neville Chi Hang, L. (2016). Evidentiary video and "Professional Vision" in the Hong Kong Umbrella Movement. Journal of Language \& Politics, 15(5), 567-588.

Keller, R. (2005). Analysing discourse: An approach from the sociology of knowledge. Forum Qualitative Sozialforschung/Forum: Qualitative Social Research, 6(3), 1-14.

Keller, R. (2011). The sociology of knowledge approach to discourse (SKAD). Human Studies, 34(1), 43-65.

Keller, R. (2013). Zur Praxis der Wissenssoziologischen Diskursanalyse [The practice of discourse analysis using the sociology of knowledge approach]. In R. Keller \& I. Truschkat (Eds.), Methodologie und Praxis der Wissenssoziologischen Diskursanalyse. Band 1: Interdisziplinäre Perspektiven [Methodology and practice of the discourse analysis using the sociology of knowledge approach. Volume 1: Interdisciplinary perspectives] (pp. 27-68). Wiesbaden: Springer VS.

Kress, G., \& van Leeuwen, T. (1996). Reading images. The grammar of visual design. London and New York, NY: Routledge.

Kress, G., \& van Leeuwen, T. (2001). Multimodal discourse: The modes and media of contemporary communication. New York, NY: Bloomsbury.

Lee, F. L., \& Chan, J. M. (2016). Digital media activities and mode of participation in a protest campaign: A study of the Umbrella Movement. Information, Communication \& Society, 19(1), 4-22.

Lee, F. L., Chen, H., \& Chan, M. (2017). Social media use and university students' participation in a largescale protest campaign: The case of Hong Kong's Umbrella Movement. Telematics \& Informatics, 34(2), 457-469.

Lee, A. L., \& Ting, K. W. (2015). Media and information praxis of young activists in the Umbrella Movement. Chinese Journal of Communication, 8(4), 376-392.

Machin, D., \& Mayr, A. (2012). How to do critical discourse analysis. London: Sage.

Ottosen, R. (2007). Emphasising images in peace journalism: Theory and practice in the case of Norway's biggest newspaper. Conflict \& Communication Online, 6(1), 1-16.

Phillips, T. (2017, August 17). Hong Kong democracy campaigners jailed over anti-China protests. The Guardian. Retrieved from https://www.theguardian. com/world/2017/aug/17/hong-kong-democracy-cam paigners-jailed-over-anti-china-protests

Rose, G. (2012). Visual methodologies. An introduction to researching with visual materials. London: Sage.

Royce, T. D., \& Bowcher, W. L. (Eds.). (2007). New directions in the analysis of multimodal discourse. Mahwah: Erlbaum.

Sikov, E. (2010). Film studies. An introduction. New York, NY: Columbia University Press.

Tufekci, Z. (2017). Twitter and tear gas: The power and fragility of networked protest. New Haven, CT, and London: Yale University Press.

Villi, M. (2007). Mobile visual communication: Photo messages and camera phone photography. Nordicom Review, 28(1), 49-62.

Vis, F., Parry, K., Manyukhina, Y., \& Evans, L. (2013). Twitpic-ing the riots: Analysing images shared on Twitter during the 2011 UK riots. In K. Weller, A. Bruns, J. Burgess, M. Mahrt, \& C. Puschmann (Eds.), Twitter and society (pp. 385-398). New York, NY: Peter Lang.

Wetzstein, I. (2011). Konstruktive Konfliktbearbeitung in der qualitätsjournalistischen Auslandsberichterstattung [The constructive handling of conflicts in quality-journalistic international news coverage]. Wiesbaden: VS Verlag.

Wetzstein, I., \& Huber, B. (2016). Debating "alternative" gender identities: The online discourse triggered by 2014 Life Ball advertising posters. International Journal of Communication, 10(1), 432-450.

Zhu, Q., Skoric, M., \& Shen, F. (2017). I shield myself from thee: Selective avoidance on social media during political protests. Political Communication, 34(1), 112-131. 


\section{About the Author}

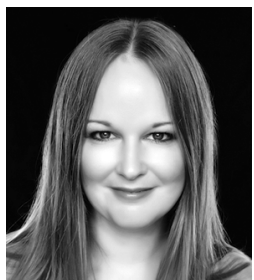

Irmgard Wetzstein (PhD) is a Senior Lecturer at the Department of Communication/University of Vienna, a former Fulbright Visiting Professor at the School of Journalism and Mass Communication at the University of Minnesota, and a trained mediator and conflict manager. Her research and teaching activities focus on social media and communication, advertising and strategic communication, visual communication as well as journalism and conflict communication. 
Annex

All of the following exemplary Twitter links are public and freely accessible.

1 https://twitter.com/Kaandeev/status/516538840970821632

2 https://twitter.com/prepperknowhow/status/517481223351181312

3 https://twitter.com/storiebox/status/516244616488955904

4 https://twitter.com/doublewingble/status/521237645989404672

5 https://twitter.com/GuardianWitness/status/516913040676372480

6 https://twitter.com/FraserLi1/status/517631574804676609

7 https://twitter.com/jimsciutto/status/518013187464384512

8 https://twitter.com/stephenmengland/status/517362285413560321

9 https://twitter.com/prashantrao/status/529054701418188802

10 https://twitter.com/YushanVentures/status/523869306132508673

11 https://twitter.com/donplusn/status/522415659800489984

12 https://twitter.com/SputnikInt/status/539107885511229441

13 https://twitter.com/WatchGirllnTown/status/523044173012692993

$14 \mathrm{https} / / /$ twitter.com/GroundTruth/status/517312956829540352

15 https://twitter.com/BUZZCLOUDTWEETS/status/532922690542129154

16 https://twitter.com/FromCaserta/status/524397415076335616

17 https://twitter.com/Ancient_Warrior/status/542748194267230208

18 https://twitter.com/queen_for_a_day/status/543406180471955456

19 https://twitter.com/umbrellarthk/status/524576118448197632

20 https://twitter.com/yessir_risa/status/527789755962900480

21 https://twitter.com/ivanlivic/status/527315328213061635

22 https://twitter.com/AlamyNews/status/542634072670220288

23 https://twitter.com/NOsurenderingNO/status/533894532828385280

$24 \mathrm{https} / / /$ twitter.com/kingwine4698/status/533189205266935809

25 https://twitter.com/donplusn/status/527482783208206336

$26 \mathrm{https} / / /$ twitter.com/ElaineToHK/status/517275008855318528

27 https://twitter.com/chrisdavis31/status/539465761748942848

28 https://twitter.com/mishfit23/status/536469158800527360

29 https://twitter.com/MSULaw/status/535137069937721346

$30 \mathrm{https}: / /$ twitter.com/brandonyvr/status/517571089812451328

31 https://twitter.com/hlrecord/status/520930168052129793

32 https://twitter.com/TunstallAsc/status/544408361081790464

33 https://twitter.com/sdmoores/status/540523002689900545

34 https://twitter.com/citypatarantola/status/543534951086698496

35 https://twitter.com/wallerpan/status/543627495061200899

$36 \mathrm{https} / / /$ twitter.com/hashtag/hongkongprotests?vertical=default\&f=images

37 https://twitter.com/reporterphoenix/status/540233112350105601

38 https://twitter.com/Bill_GP/status/522947325568618496

39 https://twitter.com/masanaminori/status/539187457179152384

$40 \mathrm{https}: / /$ twitter.com/anadoluimages/status/523144301065564160

41 https://twitter.com/friendsoftibet/status/523768353463087104

42 https://twitter.com/DevicaKong/status/522571589456375808

43 https://twitter.com/fauziah_ibrahim/status/518039501378043904

44 https://twitter.com/SexenioGTO/status/523625061748858882

45 https://twitter.com/YahooSG/status/526557928023539712

46 https://twitter.com/donplusn/status/525657247750176768

$47 \mathrm{https}: / /$ twitter.com/jfung1/status/528430310728294400

48 https://twitter.com/alanlambodublin/status/517056272634359808

49 https://twitter.com/Dispropoganda/status/523215271993946112

50 https://twitter.com/isaacaranjuez/status/524669384238039041

51 https://twitter.com/middleburgh/status/526315411579478016

52 https://twitter.com/Aoibaris/status/524581486066794497 


\section{COGITATIO}

53 https://twitter.com/donplusn/status/525931616682516483

$54 \mathrm{https} / / /$ twitter.com/MoveDemocracy/status/544510301161680898

$55 \quad$ https://twitter.com/isaacchongwai/status/543766409201717248

56 https://twitter.com/thehongkongkid/status/527343411716571137 